\title{
Monte Carlo Simulation for Distance and Absorbed Dose Calculations in a PET-CT Facility by Using MCNP-X
}

\author{
Huseyin Ozan TEKIN ${ }^{1}$ and Umit KARA ${ }^{2}$ \\ 1. Vocational School of Health Service, Radiotherapy Department, Uskudar University, Istanbu 34672l, Turkey \\ 2. Vocational School of Health Service, Medical Imaging Department, Suleyman Demirel University, Isparta, Turkey
}

\begin{abstract}
Modern PET/CT clinics consist of a scanner room housing the double gantry PET/CT unit and a control area. These areas should be considered as a parts of facility that must be protected. The scanner room, quiet rooms, and frequently the hot lab require structural shielding to protect staff and personnel in surrounding areas. The $511 \mathrm{keV}$ photons from the PET positron emitting isotopes are the source term for the quiet rooms and the hot lab, while both the $511 \mathrm{keV}$ photons and the multi-energetic spectrum of $\mathrm{x}$-rays from the CT unit must be considered for the scanner room. In this study, we have developed a 3D (three dimensional) model of PET-CT scanner room by using MCNP-X (version 2.4.0) Monte Carlo code. We considered the distance factor in PET-CT facility for the amount of exposure radiation dose received by staff. In terms of exposed radiation dose of staff, we obtained the well agreement on distance factor calculations by using Monte Carlo method. We achieved a good agreement between MCNP-X results and clinical experimental results.
\end{abstract}

Key words: Monte Carlo, PET-CT, radiation protection.

\section{Introduction}

PET (positron emission tomography) is widely used for diagnosis, staging, and follow- up of various malignancies. It has been studied in the evaluation of various tumor kinds such as lung cancer, head and neck tumors, colon and rectal cancer, lymphoma, malignant melanoma, breast cancer, thyroid cancer, over gynecologic tumors such as endometrial and cervical cancer, primary or metastatic brain tumors. However, MR (magnetic resonance) and computed tomography have superior capabilities on monitoring of anatomic changes for diagnosis and follow-up the cancer [1]. Although PET has a strong abilities on follow-up process in oncological studies, there is need for different requirements on some issues such as certainly identification of anatomical regions. In this case, it has emerged the need to use different hybrid devices such as PET-CT. Since PET is limited by

Corresponding author: Huseyin Ozan Tekin, assist. prof. Dr., research fields: Monte Carlo simulations, medical radiation, radiation physics, medical imaging physics. weak anatomic details, a big need occured to fusion images with CT. It allows different images to be taken at the same time without moving patient. This device is capable of displaying simultaneously the properties of the two separated devices. While X-ray CT (computarized tomography) allows imaging the mass density of living tissues by using the external x-ray tube, PET images gamma rays directly emitted from tissues. In this case, the emission of gamma rays results from the decay of radioactive nuclei used to label a radiopharmaceutical injected to the patient. Besides the use of two different imaging devices, in fact, we see here the two different imaging methods such as transmission of x-rays emitted by CT and emission of gamma rays emitted by radionuclides and scanned by PET. This has become a very effective method and highlighted in previous scientific studies [2]. Fig. 1 shows the main structure of PET-CT device. We can see from Fig. 1 that PET modules and CT modules combined in same area. 


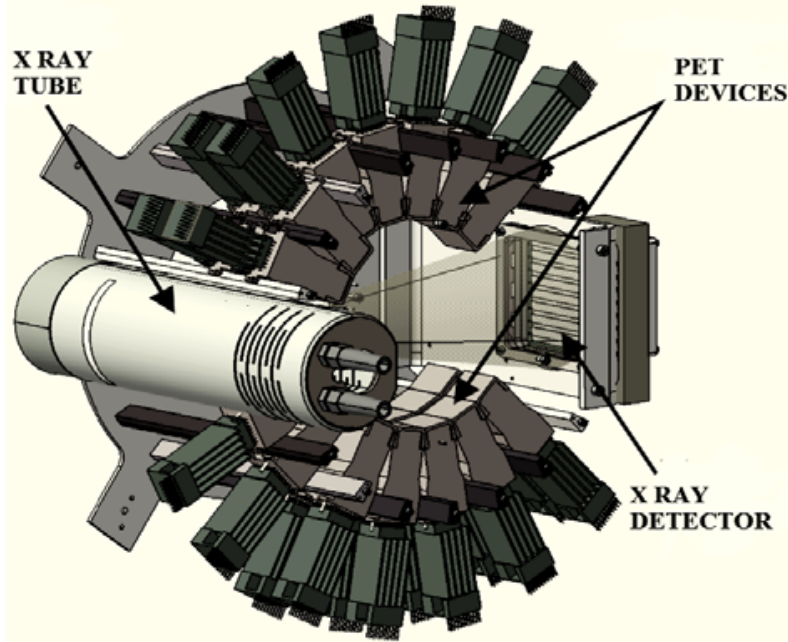

Fig. 1 A schematic view of PET-CT.

\section{Radiation from a PET-CT}

The use of PET-CT is growing rapidly because a FDG (form of glucose) labeled with fluorine-18 is such a valuable tracer for imaging changes in the uptake of glucose in body tissues, especially in cancer, and because the supply of F-18 is expanding while it combines with excellent imaging capabilities of the CT's on anatomical structure. Patients who have received radiopharmaceuticals become a source of contamination and exposure for people, who come in contact with them, such as nuclear medicine technologists, nurses, visiting relatives [3]. Some previous studies on absorbed dose radiation of nuclear medicine staff have been done in different medical research centers $[4,5]$. It is used different dosimeter types such as film, TLD (thermoluminescent dosimeter) or OSL (optically stimulated luminescence) dosimeter to record the amount of dose absorbed by the employees. To reduce the amount of dose absorbed by nuclear medicine staff there are a few issues that need to be considered. One of them which are also investigated in this study are distance factors in PET-CT facilities. It is well known that absorbed radiation dose decreases by increasing distance from the source and to optimized shielding materials in PET-CT facility [6].

In a PET-CT examination patient is exposed to radiation internally during PET administered activity F-18 and externally during CT. On the other hand, staff of nuclear medicine exposed to radiation also by handling radiopharmaceutical such as dose preparation, transport of syringe to injection room, injection of patient, measure the residual activity, handling of radioactive waste and close contact with the patient while the patient became a radioactive source. In recent years, ICRP (international commission on radiological protection) attaches importance to fundamental principles for radiation protection by considering three main subjects as justification, optimization and dose limitation [7].

The field of optimization can be evaluated under three main headings as shielding, time and distance. In this study, one of those headings such as distance factors considered in PET-CT facility. As a specific approach to radiation dose and distance factor can be described as the amount of radiation an individual receives will also depend on how close the person is to the source. It can be called as Inverse Square Law.

$$
\mathrm{I}_{1} / \mathrm{I}_{2}=\left(\mathrm{D}_{2}\right)^{2} /\left(\mathrm{D}_{1}\right)^{2}
$$

In Eq. (1), where $D_{1}$ is first distance from source, $D_{2}$ is second distance from source, $I_{1}$ is radiation intensity at $\mathrm{D}_{1}, \mathrm{I}_{2}$ is radiation intensity at $\mathrm{D}_{2}$.

\section{Summary of MCNP-X Code}

MCNP-X (Monte Carlo N-Particle eXtended), is a general purpose Monte Carlo radiation transport code for modeling the interaction of radiation with materials. MCNP-X is fully three-dimensional and it utilizes extended nuclear cross section libraries and uses physics models for particle types. MCNP-X is used for nuclear medicine, radiation physics, nuclear safeguards, accelerator applications, nuclear criticality etc. It also allows the various specific working areas about simulations. One of them is modeling the detector geometry. The detector geometry is modeled with the MCNP-X code, which allows simulating physical events occurring during the detection and registers them to build energy spectra. MCNP-X is 
also allowed for calculating the flux at a point or ring that name is F5 tally. For each source particle and each collision event, a deterministic estimate is made of the fluence contribution at the detector point [8].

Considering the geometry shown in Fig. 1 with a sectional view of PET-CT facility has been modeled by using MCNP-X simulation for efficiency calculation by using F5 tally. Physical parameters of related facility shown in Fig. 2 taken from Suleyman Demirel University PET-CT facility [9] have been modeled in MCNP-X code.

\section{Results}

Gamma radiation is the part of the electromagnetic

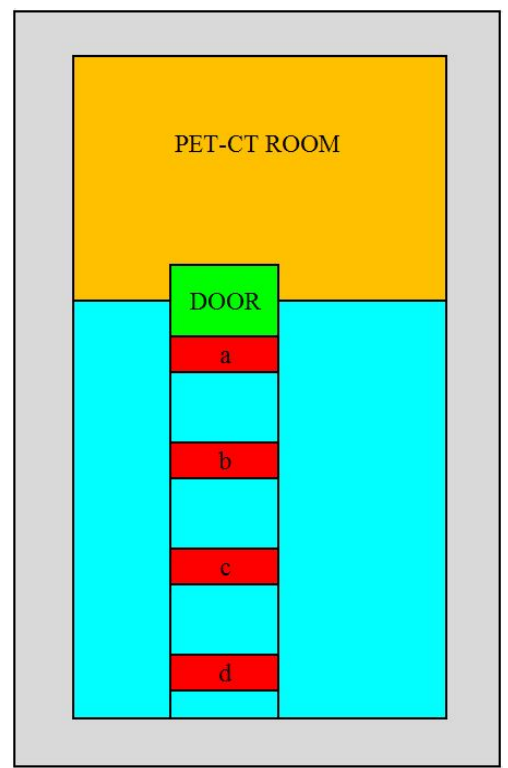

Fig. 2 Schematic representation of PET-CT facility.

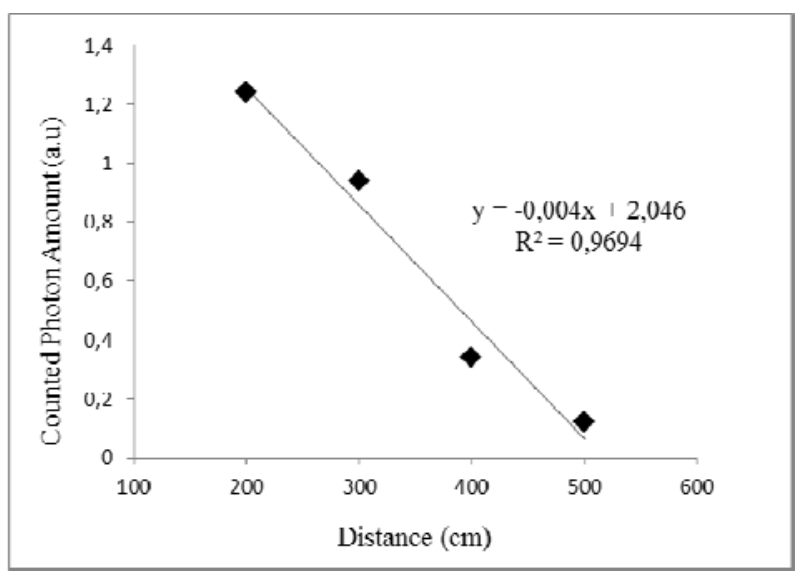

Fig. 3 Change of photon intensity with distance. spectrum. A gamma radiation ray not absorbed by the air, but its intensity decreases because it spreads out. Accordingly, the intensity changes with the inverse square of distance: it follows an inverse square law. By taking into account the distance factor some experimental calculations were made by Kara et al. 2015. They achieved a well agreement between absorbed dose and distance from facility. In this study, we modeled a PET-CT facility which emits exposure gamma radiation from area. As it's seen in Fig. 2, the F5 point detectors located on four different places $\mathrm{a}=$ $200 \mathrm{~cm}, \mathrm{~b}=300 \mathrm{~cm}, \mathrm{c}=400 \mathrm{~cm}$ and $\mathrm{d}=500 \mathrm{~cm}$ respectively. In our calculations, we achieved that radiation dose reduced by increasing distance from the facility.

\section{Conclusion}

To obtain accurate results from Monte Carlo simulations in counted photon calculations, precise modeling of the geometry and a sufficiently large number of particles are required. In this study, we simulated the dose rates in different distances. We studied the effect of distance on percentage dose rates for four different distance respectively. We achieved that Monte Carlo is very effective tool to calculate dose rates in different distances. In recent years, radiation protection of medical staff became one of most important issue that also pointed out with ICRP's annual reports. The results of this study suggested that the calculation of dose rates in Monte Carlo simulation of PET-CT could provide useful information about the effect of radiation and its effects on staff. Also Monte Carlo simulation can make possible to future studies for various subjects on medical radiation such as shielding factor and shielding materials.

\section{References}

[1] Kapoor, V., McCook, B., and Torok, F. 2004. "An Introduction to PET-CT Imaging.” Radio Graphics Journal 24: 523-43. DOI: 10.1148/RG.242025724.

[2] Yamashita, H. 2014. "Clinical Value of Whole-Body 
PET/CT in Patients with Active Rheumatic Diseases.” Arthritis Research \& Therapy 16: 423. DOI: 10.1186/S13075-014-0423-2.

[3] Yin, H. S., and Douglas, R. S. 1992. "Radioactive Contamination in Hospitals from Nuclear Medicine Patients." Health Phys 62 (5): 462-66. DOI: 10.1097/00004032-199205000-00015.

[4] Sattari, A. 2004. "Radiation Dose to the Nuclear Medicine Nurses.” Iranian Journal of Radiation Research (IJRR) 2 (2): 59-62.

[5] Nadia, H. 2012. "Evaluation of Internal Exposure of Nuclear Medicine Staff Using In-Vitro and Organ Dose Calculation Methodologies.” IJRRAS 11 (3): 536-45.
[6] Peet, D. J. 2012. "Radiation Protection in Fixed PET/CT Facilities-Design and Operation.” The British Journal of Radiology 85: 643-6. DOI: 10.1259/bjr/32969351.

[7] Valentin, J. 2007. "The 2007 Recommendations of the International Commission on Radiological Protection (ICRP).” Presented at the Annals of the ICRP. Published for the International Commission On Radiological Protection. DOI:10.4016/13844.01.

[8] Pelowitz, D. B. 2005. MCNPX USER'S MANUAL version 2.5 LA-UR-05 2675. USA: Los Alamos National Laboratory (LANL).

[9] Kara, U., Tekin, H. O., and Akkurt, I. 2015. "Radiation Protection in PET Room.” Acta Physica Polonica A. 128. DOI: 10.12693/APHYSPOLA.128.B-375. 